\title{
INVESTIGACIONES
}

\section{Aprendizaje autónomo y plataformas digitales: el uso de tutoriales de YouTube de jóvenes en Ecuador ${ }^{*}$}

\author{
Autonomous learning and digital platforms: \\ use of YouTube tutorials of young people in Ecuador \\ Edison J. Padilla ${ }^{a}$, Gladys I. Portilla ${ }^{a}$, Manuel Torres ${ }^{b}$ \\ ${ }^{a}$ Universidad Nacional de Educación. \\ edison.padilla@unae.edu.ec,gladys.portilla@unae.edu.ec \\ ${ }^{b}$ Universidad Estatal Península de Santa Elena. \\ mtorres@upse.edu.ec
}

\begin{abstract}
Este artículo muestra parte de los resultados de una investigación enfocada a la posible aplicación de competencias transmedia relacionadas con el YouTube en los procesos formales de enseñanza-aprendizaje, mediante una investigación mixta basada en la observación participante, cuestionarios, entrevistas, talleres y diarios de uso mediático. Los principales resultados están enfocados en el uso de tutoriales de YouTube como recursos para la generación de conocimientos educativos formales e informales, estos incluyen la creación y publicación de videos en este mismo portal, ofreciendo un compendio de posibles acciones para gestionar el aprendizaje autónomo, desde la función tutorial docente. Una de las principales conclusiones se enfoca en que los tutoriales de YouTube son un medio efectivo para satisfacer las necesidades escolares fuera del contexto escolar.
\end{abstract}

Palabras claves: enseñanza, competencias transmedia, función tutorial, recurso educativo.

\begin{abstract}
This article shows part of the results of an investigation focused on the possible application of transmedia skills related to YouTube in the formal teaching-learning processes, through a mixed investigation based on participant observation, questionnaires, interviews, workshops and daily media use. The main results are focused on the use of YouTube tutorials as resources for the generation of formal and informal educational knowledge, these include such as the creation and publication of videos in this same portal, offering a summary of possible actions to manage autonomous learning, from the teaching tutorial function. One of the main conclusions is that YouTube tutorials are an effective means to meet school needs outside the school context.
\end{abstract}

Key words: teaching, transmedia skills, tutorial function, educational resource. 


\section{INTRODUCCIÓN}

Este trabajo presenta algunos resultados de la investigación llevada a cabo en el proyecto "Educación Transmedia. Competencias transmedia y estrategias informales de aprendizaje de los adolescentes" , que expande en Ecuador la línea de investigación desarrollada en los proyectos: "Transalfabetismos"2 (Ministerio de Economía y Competitividad de España, 2015-2017) y "Transliteracy"3 (Programa H2020 de la Unión Europea, 2015-2018), ambos dirigidos por Carlos A. Scolari, de la Universidad Pompeu Fabra de Barcelona.

La investigación se desarrolló en dos escuelas de Ecuador de la Zonal 6 de Educación, una en la ciudad de Azogues y la otra en la ciudad de Cuenca. La investigación fue llevada a cabo por un equipo de investigadores de la UNAE con la participación de un docente y un estudiante de la Universidad de Cuenca. El objetivo fue identificar las competencias transmedia y las estrategias informales de aprendizaje desarrolladas por los adolescentes para aprender fuera del ámbito escolar, y con fines recreativos. A partir de la identificación de las competencias transmedia se diseñarán actividades didácticas orientadas a aprovechar estas competencias en los aprendizajes escolarizados. Estas habilidades en el aprendizaje informal abarcan desde la creación y distribución de contenidos en plataformas colaborativas de productos audiovisuales hasta la resolución de problemas en los videojuegos y la gestión de las redes sociales. La investigación analiza las prácticas informales que llevan a cabo los estudiantes de entre 12 a 17 años para la gestión de sus aprendizajes, en dos escuelas con diferentes contextos socioeducativos, la una es una escuela fiscal rural y la otra particular urbana.

Se partió de indagar lo que adolescentes y jóvenes intercambian en las redes sociales. Esta información es fundamental para desarrollar competencias requeridas en el contexto educativo del siglo XXI, ya que algunos modelos pedagógicos señalan la importancia de la capacidad de utilizar y comunicar el conocimiento de manera disciplinada, crítica y en escenarios de aprendizaje de la era digital (Álvarez, Pérez, Prats, Didriksson \& Quinn, 2015). En esta línea, la incorporación de tecnologías educativas transmedia en la docencia, no sólo implica considerar cuestiones tecnológicas, sino también proponer analizar y debatir de manera crítica los mensajes que se intercambian en espacios de comunicación intra e intergrupal y en medios digitales masivos. Estos últimos son los que más reflejan imágenes y narrativas que persiguen y consiguen llegar a determinados públicos y mantenerse entre las preferencias de éstos. Para el uso educativo de estas tecnologías es de utilidad conocer las opiniones y preferencias de los adolescentes y jóvenes acerca de las prácticas que desarrollan usando narrativas transmedia, y que nos muestran sus vivencias de aprendizaje en ambientes lúdicos e informales.

\section{MÉTODO O ESTRATEGIA DE ANÁLISIS}

La muestra consiste en estudiantes de una escuela de la ciudad de Azogues y otra escuela de la ciudad de Cuenca, de las cuales han participado 136 estudiantes de los niveles: octavo

\footnotetext{
https://educaciontransmedia.wordpress.com/

https://alfabetismotransmedia.org/

https://transmedialiteracy.org
} 
de Educación General Básica (8vo de EGB) con edades entre 12 y 14 años y tercero de Bachillerato General Unificado (3ro de BGU) con edades entre 16 y 17 años. Para la selección de estas escuelas se tuvo en cuenta que éstas son representativas en cuanto a la demografía de la región, pero también responden a perfiles diferenciados en función de su ubicación (rural o urbana) y de su financiamiento (público o privado). El período que han ocupado la petición de permisos a instituciones, padres y estudiantes, así como el trabajo de campo propiamente dicho se ha extendido desde noviembre de 2016 hasta abril de 2017. Posteriormente se codificó y analizó la información recolectada con el programa Nvivo11, facilitado por la UNAE, que permitió el análisis cualitativo de datos. Esta herramienta digital está dirigida a la investigación con métodos cualitativos y mixtos. Está diseñada para ayudar a organizar, analizar y encontrar perspectivas en datos no estructurados o cualitativos, provenientes de instrumentos de recolección de información como: entrevistas, encuestas con preguntas abiertas, artículos, libros, contenido de las redes sociales web y otros (QSR International, 2017).

En el análisis se identificaron las competencias transmedia de los estudiantes, objeto de la investigación, para con base en dichas competencias crear los productos didácticos de la investigación, entre los cuales se puede citar la creación de un MOOC enfocado a socializar el uso de las estrategias transmedia en la gestión del currículo ecuatoriano, un Kit para los docentes, en donde se encuentran diferentes recursos para la implementación de procesos de enseñanza-aprendizaje recurriendo a las competencias transmedia de los estudiantes.

A continuación, se especifica las técnicas utilizadas para la recolección de datos en las diferentes unidades educativas que han participado como muestra. La técnica de la observación participante, utilizada en diferentes etapas de la investigación, permitió obtener información directa y experiencial de las instituciones seleccionadas para el estudio, en torno al uso de tecnologías transmedia, la sociabilidad, los valores compartidos, las formas de organización y las narrativas de los adolescentes en red.

Para mapear las características sociológicas y demográficas de los adolescentes que participaron en la investigación se utilizaron cuestionarios, ya sea en línea o presenciales. Las entrevistas con actores relevantes detectados en las fases previas facilitaron la comprensión de aspectos como las estrategias individuales de aprendizaje en línea o acceder a narraciones acerca de sus propias experiencias. Estas entrevistas incluyeron historias de vida y tuvieron un carácter autobiográfico respecto a las prácticas mediáticas.

Los talleres fueron una instancia intermedia de recolección de datos entre los cuestionarios de carácter general y las entrevistas individuales en profundidad. A través de los talleres, ya sean orientados a los videojuegos o la narrativa, se obtuvieron datos sobre valores compartidos, adquisición de competencias, estrategias grupales de aprendizaje en línea, procesos de creación, resolución de problemas, etc. El equipo realizó dos talleres en cada unidad educativa y en cada nivel, que participaron en la investigación gestionando un total de ocho talleres. Los talleres tuvieron un carácter lúdico-creativo y se basaron en técnicas gamestorming (Gray, Brown \& Macanufo, 2012) y otras actividades participativas destinadas a facilitar los intercambios con los adolescentes.

Se recurrió a la escritura, por parte de los adolescentes y jóvenes participantes, de diarios de uso y consumo mediático, como técnica complementaria para ampliar la información obtenida a través de los talleres y entrevistas. Los diarios brindaron información sobre las tiempos, frecuencias, repitencias, uso y gestión de diferentes recursos transmedia. 
A través de todas estas técnicas se pretende crear un puente para acercar las prácticas educativas formales a la realidad cotidiana de adolescentes y jóvenes inmersos en las redes sociales y la comunicación digital interactiva y contribuir a romper la barrera que existe entre los docentes y estudiantes en lo referente al consumo mediático, con el fin de potenciar prácticas docentes contextualizas en la era digital y sus recursos y narrativas transmedia.

La investigación contó con diversas actividades participativas, desarrolladas en las unidades educativas Luis Cordero de la ciudad de Azogues y Técnico Salesiano de la ciudad de Cuenca, tanto las que estaban previstas como las que surgieron durante la realización del trabajo de campo, descritas a continuación:

- Desarrollo de una sesión de información para obtener el consentimiento de padres de familia en las dos unidades educativas con un total de 136 participantes.

- Desarrollo de una sesión de información para obtener el consentimiento de estudiantes en las dos unidades educativas con un total de 142 participantes.

- Implementación de una encuesta de usos mediáticos a los estudiantes que presentaron los dos consentimientos de participación de las dos unidades educativas, con un total de 136 participantes.

- Implementación de talleres de cultura participativa en cada unidad educativa y por niveles (acotado de EGB y tercero de BGU), desarrollando un total de cuatro talleres con 74 participantes.

- Implementación de talleres de videojuegos en cada unidad educativa y por niveles (acotado de EGB y tercero de BGU), desarrollando un total de cuatro talleres con 62 participantes.

- Aplicación de diarios de uso y consumo mediático a estudiantes destacados en la exposición de competencias transmedia en cada uno de los talleres, tanto de videojuegos como de cultura participativa, con un total de 19 participantes.

- Desarrollo de entrevistas personales a estudiantes destacados en la exposición de competencias transmedia en cada uno de los talleres, tanto de videojuegos como de cultura participativa, a un total de 19 participantes.

En la siguiente sección se detalla la información principal que se ha podido extraer durante la implementación de los talleres y entrevistas analizadas con el programa Nvivo11.

\section{DESARROLLO}

\section{1. ¿CÓMO Y PARA QUÉ ACCEDEN?}

De los datos encontrados en las entrevistas se puede diferenciar varias formas en las que los jóvenes acceden a los tutoriales de YouTube. El acceso es un indicador clave para el análisis de las competencias transmedia. Para el análisis de este indicador se han considerado los medios y finalidades de acceso.

Para Scolari, Winocur, Pereira \& Barreneche (2018), lo transmedia se puede gestionar en una extensa variedad de medios, géneros y formatos, en este sentido, en este apartado de fundamentación nos referiremos a los medios como los diferentes dispositivos aplicaciones y buscadores, utilizados en las diferentes finalidades de la gestión de la información digital 
que, los adolescentes y jóvenes participantes en esta investigación, utilizaban para acceder a los tutoriales de YouTube.

En cuanto a los dispositivos, para Gutiérrez \& Gómez (2015), dentro de las tecnologías de la información y comunicación los más comunes son la computadora, teléfonos móviles y tablets. En correspondencia, se enfocó la investigación, en los dispositivos que más utilizan. Según las entrevistas a profundidad, entre los más usados se encuentran la computadora y los teléfonos móviles. Por ejemplo, en cuanto al uso de la computadora, un participante dijo: "mi amiga María Paz me enseñó a poner WhatsApp en la computadora, entonces eso también tengo, pero muy poco abro" (1054, entrevista, 2017). En cuanto al uso del celular: "No, o sea en el celular, la aplicación que ya está lista para editar el video, y para fotos utilizo PicsArt, pero esa es para celular" (130, entrevista, 2017). En los diarios de uso mediático se evidenció el poco uso de tablets.

En relación con las aplicaciones, como resultado de las entrevistas se identificó una gran cantidad de variedad de aplicaciones específicas para celular y para computadora, entre las que más se mencionan están YouTube, Facebook, Messenger, WhatsApp, Snapchat, LidowPhoto, Photo Warner, Piano tiles, Musical.ly, PicsArt, Ask, Wattpad, TubeMate, Collage, BeFunky, Movie Maker, Pinterest, Gmail, Power director, Instagram, VideShow, RePelis, entre otras. Así se refiere un de los participantes en torno a las aplicaciones que usa: "Si, por lo general tengo prendido la Tv, la compu y mi celular, en la televisión pongo Tele hit, que dan solo música, veo Facebook y mi celu para WhatsApp y Snapchat y así" (130, entrevista, 2017).

Si bien, en cuanto a los buscadores no existe tanta variedad como en las aplicaciones, entre los principales tenemos a YouTube, principalmente como gestor de búsqueda musical, y para entretenimiento en general; Google, Wikipedia, Sipeliculas, entre otros. Uno de los participantes relata como hace sus búsquedas: "Ya. Haber pongo en la pestaña de Google 'buscar biografía' entonces busco. [Busca biografía sobre su cantante favorito en Wikipedia]" (105, entrevista, 2017).

La finalidad del acceso a los recursos transmedia desde los diferentes medios determina el tipo de sujeto y el nivel de competencias que ha alcanzado dicho sujeto, según Scolari et al. (2018). El consumidor de medios se ha convertido en un desarrollador de competencias que comprenden la creación y socialización de recursos y contenidos en entornos digitales. En complemento para Bolivar, Chaverra \& Monsalve (2015), según la finalidad del uso de las diferentes aplicaciones se puede clasificar a los usuarios dentro de la Web 1.0 o dentro de la Web 2.0, en donde por un lado, a la Web 1.0 se considera únicamente con la finalidad de divulgación de contenido, y por otro lado la Web 2.0, con mejores características de software, permite a los usuarios trabajar en blogs, wikis, enfocándose preferentemente en la creación de contenido, en el trabajo colaborativo y la socialización del conocimiento. Bajo estas dos perspectivas están englobadas las finalidades de acceso a los recursos transmedia de los participantes de la investigación.

Por un lado, considerando únicamente lo que para Bolivar et al. (2015) considera Web 1.0, los entrevistados acceden a los recursos transmedia principalmente para entretenerse con información audiovisual y de lectura como por ejemplo buscar videos musicales y series en YouTube y lectura de libros digitales “... también "Hola soy Germán” que es comedia; en cambio esta página es comedia, o sea hace bromas; la vida trágica los

105: Código del entrevistado. Todas las referencias a las entrevistas tienen un código para resguardar su identidad. 
convierte en broma o las mentiras... y "Enchufe.tv" esos son los que más me gustan de YouTube, mis "youtubers" favoritos" (105, entrevista, 2017). "Si por ejemplo yo, hay una aplicación que se llama Wattpad, y yo en esta empecé a ver libros de 50 sombras de Grey y vi también la película, entonces esa creo que es la que me gusto bastante, pero la película no se compara con el libro" (130, entrevista, 2017).

Los adolescentes y los jóvenes han desarrollado la capacidad de buscar información y aprender de forma autónoma, si bien, generalmente lo hacen con fines recreativos o para satisfacer necesidades de su vida personal. "Sí, creo que sí, creo que, en la Yuya, porque creo que ella me gusta y te aconseja como hacer, como maquillarte como vestirte" (130, entrevista, 2017). Estos hábitos tienen el potencial de ser llevados al mundo de las tareas formales de su escolaridad, de hecho, esto está sucediendo, solo que a espaldas de los docentes. "A veces cuando quiero ver imágenes o deberes. Aunque me gusta más este por me explica con el video y no tienes que estar leyendo todo" (92, entrevista, 2017).

Por otro lado, existen otras competencias encontradas en la investigación que están más en la lógica de la Web 2.0, caracterizada Bolivar et al. (2015), entre ellas la comunicación interactiva, síncrona y asíncrona, cuestionarios interactivos de distracción y enfocados al estudio, consulta y compartición de recursos generales y educativos, creación y edición de videos y fotos, edición de características de contenido multimedia, etc. Así lo demuestra algunos relatos de los estudiantes participantes: "A veces hago eso, por ejemplo, capturo una imagen y subo y escribo lo que estoy sintiendo ese rato" (98, entrevista, 2017). "Uso una aplicación para hacer video y editar videos y poner efectos..." (130, entrevista, 2017). "por ejemplo, para, para ahorita los exámenes busco simuladores (simuladores del SNNA) eso" (130, entrevista, 2017).

A modo de resumen, en cuanto a los modos de acceso y finalidades, los adolescentes y jóvenes, cuyas competencias transmedia han sido analizadas, acceden a los medios y contenidos multimedia por diferentes dispositivos, entre los principales la computadora y el celular. En cuanto a la finalidad del acceso se categorizó según las características de la Web 1.0 evidenciando competencias como el acceso a información audiovisual y de lectura, y en cuanto a la Web 2.0, se encontró que han desarrollado competencias para editar, producir y compartir, de forma interactiva, recursos multimedia, creando de esto modo narrativas y vivencias de aprendizaje transmedia. Como un producto de la investigación, basado en el análisis de los recursos obtenidos de las diferentes actividades, se crearon competencias transmedia que podrían utilizarse para la gestión de la enseñanza-aprendizaje escolarizada, entre las que se refieren a este campo específico de análisis se tiene:

- Capacidad para usar diferentes dispositivos, aplicaciones y buscadores.

- Capacidad para buscar contenidos audiovisuales y otros transmedia mediante diversos buscadores, plataformas o aplicaciones.

- Capacidad para autorregular el consumo mediático por medio del pensamiento secuencial.

- Capacidad para gestionar, a través de Internet, las fuentes de información con base en información relacionada con preferencias de entretenimiento.

- Aprender con recursos transmedia.

- Capacidad para generar la motivación intrínseca capacidad para motivarse a través de recursos transmedia en la utilización de otros recursos.

- Capacidad para hacer multitarea (eficientemente). 
- Capacidad para compartir recursos transmedia.

- Capacidad de desarrollar trabajo colaborativo mediante el uso de diversas plataformas digitales y las redes sociales.

- Capacidad para identificar y clasificar contenidos para su reutilización offline.

- Capacidad de crear recursos transmedia.

- Capacidad de comunicarse de forma interactiva mediante recursos transmedia.

\section{2. ¿CÓMO (TUTORIALES DE YOUTUBE) Y PARA QUÉ (APRENDIZAJE AUTÓNOMO) USAN LA INFORMACIÓN DEL YOUTUBE?}

Como se mencionó en el apartado anterior los niños y jóvenes utilizan los recursos transmedia para gestionar su aprendizaje autónomo, ya sea por aficiones personales o por necesidades escolares. En este apartado se abordará el uso de los tutoriales de YouTube en estos dos sentidos, aficiones personales o necesidades escolares.

El recurso YouTube, según Ruiz \& Escurra (2013), se trata de una plataforma que permite a sus usuarios no solo observar videos si no también compartirlos de manera gratuita, además, en cuanto al uso de la plataforma, aseguran que dentro de su investigación un 48,6 $\%$ de los participantes indicaron que utilizan YouTube para desarrollar tareas escolares.

Centrando la atención en la necesidad personal de aprender algo nuevo se ha podido evidenciar situaciones en las que se accede a tutoriales de YouTube para aprender alguna estrategia de juego, encontrar consejos para mejorar su apariencia personal y de sus pertenencias, mejorar sus aptitudes, crear y editar videos, uso de dispositivos, entre otros. Así relata uno de los participantes en este proyecto. "También el de "Piano tiles". [En una pestaña de YouTube busca "cómo hacer lento piano tiles"] A veces en YouTube he puesto y me explicaron que debo hacer con un papel, entonces hay que poner los dedos "asî" y ahí, para. Y es verdad, y es efectivamente" (105, entrevista, 2017). "En YouTube... yo... mis tres "youtubers" favoritos es "Yuya" que ya enseña como maquillarse, los portes, la nueva decoración de mi cuarto cosas así. También "Hola soy Germán" que es comedia; en cambio esta página es comedia, o sea hace bromas; la vida trágica los convierte en broma o las mentiras..." (105, entrevista, 2017).

Al centrar la atención en el desarrollo de actividades escolares se identificó diferentes tipos de circunstancias y situaciones, entre las principales tenemos el refuerzo de contenidos trabajados en las aulas de clase, ayuda en proyectos escolares, búsqueda y aprendizaje de conocimientos relacionados con sus asignaturas, búsqueda de explicaciones más sencillas de temas desarrollados en sus aulas de clase, entre otros. Estas son algunas versiones de estas competencias en torno a aprendizajes escolares: "Ya, haber yo no sé prestar mucha atención a clases (risas), entonces yo vengo y pongo tutoriales de un tema cualquiera, por ejemplo. [En el computador abre YouTube y busca "vectores en matemáticas"] siempre pongo ahí y me dan videos, o sea me dan clases virtual, entonces eso es lo que más utilizo..." (105, entrevista, 2017). "Es que usted entra... póngase o sea yo estudio Mecatrónica y no sé algo, entonces pongo tal cosa como se hace y ahí sale una lista, entonces voy viendo cómo se hace y cada vez voy conectando con un programa con otro programa. Voy viendo de poco en poco..." (35, entrevista, 2017). "Pero, o sea, sí se alcanza a comunicar bastante con las redes sociales con los profesores y ese tipo de cosas; y si no, aquí mismo con, hay tutoriales que enseñan a hacer más lo que es matemáticas y ese tipo de cosas y explican bastante. Es hasta más simple" (65, entrevista, 2017). 
En el uso de tutoriales de YouTube se logró identificar dos situaciones de mucho interés para su uso en contextos escolares. La primera se refiere al uso de YouTube, no solo del video como tal, sino a su capacidad de interactividad, que brindan posibilidad de hacer comentarios al pie de los videos para pedir aclaraciones si el caso lo amerita: "Si tienes una duda en el video ¿cómo lo intentas resolver esa duda? Tal vez comentarle en los comentarios..." (105, entrevista, 2017). Una segunda situación se refiere a que, incluso teniendo una persona de referencia para consultarle sus dudas, aun así, prefieren revisar tutoriales de YouTube: “....siempre pongo ahí y me dan videos, o sea me dan clases virtual, entonces eso es lo que más utilizo; y, como segunda opción cuando mami está en casa ella me explica... entonces..." (105, entrevista, 2017).

Resumiendo, sobre el cómo y para qué utilizan tutoriales de YouTube, los adolescentes y jóvenes utilizan estos tutoriales para gestionar su aprendizaje autónomo enfocado tanto a sus necesidades personales de aprendizaje como a los aprendizajes formales, dentro y fuera de los centros educativos. Entre los principales aprendizajes orientados por sus necesidades personales se identificaron el aprender estrategias de juego, obtener consejos personales, mejorar sus aptitudes, crear y editar videos y uso de dispositivos. Para los aprendizajes formales recurren a tutoriales para refuerzo de contenidos, desarrollo de proyectos escolares y búsqueda de explicaciones más sencillas sobre temas de estudio. Con base en los resultados del análisis de las habilidades transmedia de los adolescentes y jóvenes, se han identificado competencias transmedia en el campo de los aprendizajes formales, estás son:

- Capacidad para usar diferentes dispositivos, aplicaciones y buscadores.

- Capacidad para buscar contenidos audiovisuales y otros transmedia mediante diversos buscadores, plataformas o aplicaciones.

- Aprender mediante-recursos transmedia.

- Capacidad para analizar contenido multimedia.

- Capacidad de comunicarse mediante recursos transmedia.

En su investigación, Cancelas \& Herrera (2014), muestran un conjunto de herramientas dentro de YouTube, que están orientadas al uso educativo las competencias transmedia, entre las más usadas está YouTube for schools, que es una herramienta destinada a la enseñanza e impide el acceso a otros videos fuera del contexto educativo, en esta herramienta existen listas de videos por asignaturas permitiendo una búsqueda ágil y dinámica. En este sentido, de las competencias observadas en esta investigación, se puede ver el potencial de la herramienta YouTube for schools, la cual puede ser utilizada por los docentes en la gestión de sus procesos de enseñanza-aprendizaje y optimizar las competencias transmedia que los estudiantes ya poseen.

El potencial de competencias transmedia que los niños pueden implementar no solo tiene como fundamento esta investigación que está enfocada en adolescentes y jóvenes de entre 12 y 17 años. Según León (2018), quien desarrolló su investigación en niños de entre 7 y 11 años, asegura que los niños no únicamente son consumidores activos de videos de YouTube, sino que sus competencias están enfocadas a la producción, edición y compartición de videos en la plataforma YouTube, de esta manera se puede complementar y confirmar los altos grados de competencias transmedia que tienen los niños y jóvenes, especialmente en el manejo de la herramienta YouTube. 


\section{3. ¿QUÉ, CÓMO Y PARA QUÉ PUBLICAN?}

Según los datos obtenidos en esta investigación, la principal herramienta transmedia que utilizan los adolescentes y los jóvenes es el YouTube. Los resultados de la investigación dan cuenta de que publican tutoriales y eventos personales en redes sociales, de forma cotidiana y con diferentes fines. A través de esta plataforma acceden a tutoriales y aprenden, de forma autónoma y según sus propios intereses y ritmos, lo que necesitan para jugar videojuegos, resolver dudas en torno a temas relacionados con la personalidad y vida íntima, como la apariencia, la moda, la afectividad, etc.

Pasan del acceso y consumo de información transmedia a la producción, en la lógica y la dinámica de la web 2.0, creando videos tutoriales sobre hallazgos en torno a los videojuegos y otros temas de entretenimiento y de interés personal. De este modo, alimentan las redes, en la dinámica del conectivismo (Siemens, 2004), pues como usuarios asiduos, entienden el valor de compartir lo que saben ya qué podría ser de utilidad para alguien.

La escuela alberga en sus aulas a chicos que aprenden de forma autónoma, llevando el rol tutorial en el aprendizaje a situaciones inéditas; han desarrollado las capacidades para aprender y compartir, creando oportunidades para que otros aprendan con los videos tutoriales en YouTube. Han convertido el rol tutorial para el aprendizaje, de tanta actualidad en el mundo educativo, como lo demuestran algunos estudios (Rodríguez, Hinojo y Ágreda, 2017; Velarde, Dehesa, López y Márquez, 2017) en una cultura juvenil transmedia sin precedentes, dentro y fuera de los sistemas de aprendizaje escolarizado. Son chicos que han aprendido a aprender, de forma autorregulada, conectados y en la ubicuidad, para entretenerse y configurar y vivir sus mundos personales, cotidianos y domésticos del mundo digital.

De este modo se ha instaurado en los aprendizajes informales, no escolarizados los aprendizajes autónomos, ubicuos y altamente efectivos. Los adolescentes y jóvenes están aprendiendo por sus propios medios y con sus propias culturas educativas, en las que predomina la tutorización, tanto para el acceso como para la producción y publicación de recursos de aprendizaje transmedia.

La escuela tiene el desafío de llevar a esos escenarios transmedia, autónomos y ubicuos los aprendizajes escolares, curriculares.

\section{4. ¿CÓMO USAR EDUCATIVAMENTE LAS COMPETENCIAS TECNOLÓGICAS DESARROLLADAS A TRAVÉS DEL YOUTUBE?}

Los efectos nocivos de los videojuegos, vistos como un problema de gran preocupación para la salud (Salas, 2017; Robaina, Jenaro, González, Martín y Poy, 2013), pueden ser revertidos al darles un viraje educativo, aprovechando su potencial didáctico para el aprendizaje escolar, como lo demuestran varios autores (Betancur et al., 2014; Badia et al., 2015; Marín, 2015). Entre las principales características y funcionalidades de las competencias tecnológicas desarrolladas a través del YouTube, que podrían ser usadas para los aprendizajes escolarizados están: lo lúdico, la ubicuidad, la interactividad y la tutorización en tiempos reales de aprendizaje.

La interactividad y lo lúdico son características de los videojuegos que pueden ser aprovechados para desarrollar aprendizajes escolares tales como Historia, Lengua y 
Literatura, Matemática, Ciencias Naturales entre otros campos curriculares, de forma interactiva, colaborativa y entretenida, a través de las, tramas, personajes, escenarios y elementos de las narrativas transmedia. Hay un sinnúmero de herramientas digitales de software libre, intuitivas y con gran potencial educativo, pero para que lleguen al aula es preciso que los docentes reconozcan, comprendan e incorporen las competencias transmedia de los estudiantes, lo que implica que los docentes deben desarrollar las competencias digitales para el uso de dichas competencias en los aprendizajes.

Los videojuegos implican habilidades cognitivas tales como: identificar la secuencialidad en la trama, la caracterización de los personajes, la búsqueda de estrategias para avanzar y ganar el juego, afrontar el desafío y la incertidumbre de aprender de forma autónoma, entre otras. Estás habilidades tienen gran potencial educativo para aprender en la escuela en una lógica superadora de los aprendizajes memorísticos.

Un elemento educativo clave de los videojuegos es el rol protagónico del jugador. Los aprendizajes auténticos son aquellos que involucran al estudiante, de tal forma que se vuelve el principal actor de sus aprendizajes, al gestionar recursos, apoyo y escenarios para que sucedan los aprendizajes que son queridos por los estudiantes.

La efectividad de la tutorización a través de los multimedia se debe, entre otras condiciones, a que ocurre en el tiempo real en el que ocurren los aprendizajes, se puede a recurrir a tutoriales y consultas interactivas en el momento en el que se aprende, sobre la marcha. Para aprovechar esta característica de las competencias transmedia los docentes precisan incursionar en la creación entornos virtuales, tales como Edmodo, Moodle, entre otras permitiendo que los recursos multimedia se conviertan en un aliado estratégico para aprovechar el potencial de los tutoriales de videos, foros, chats y otros recursos transmedia.

Ir de los aprendizajes dentro del aula escolar, presencial y dirigido únicamente por el docente implica ir del unilateral aprendizaje al blended learning, del aula tradicional al aula invertida. Los docentes deben pasar de ser sólo consumidores a creadores de recursos multimedia adecuados a las necesidades y enseñanza-aprendizaje específicos. Los docentes tienen en sus aulas a expertos en audiovisuales y búsqueda de información. Los estudiantes podrían apoyar, desde sus competencias tecnológicas, en el diseño de los entornos y recursos transmedia educativos, para el aprendizaje del grupo.

Las herramientas multimedia son altamente intuitivas, y esto constituye una condición determinante para el uso de estas, por parte de los docentes sin formación específica en este tipo de herramientas tecnológicas. Y lo que no se logra de forma intuitiva, se puede lograr a través de los mismos videos tutoriales, con los que se puede aprender a hacer, un video, crear un aula virtual, crear un blog, crear una página web, etc.

La formación de docentes podría orientarse a aprovechar sus mismos espacios y desafíos de desempeño, en función de necesidades específicas de la profesión. En la era digital, para este tipo de formación es necesario recurrir a las tecnologías digitales, que tienen el potencial educativo de aprender a usarlas a través de los mismos recursos tecnológicos. Se puede aprender a hacer videos tutoriales recurriendo a los mismos videos tutoriales para hacer tutoriales.

Para fomentar el uso educativo de los recursos transmedia, por parte de los profesores, es de mucha ayuda disponer de un Kit con competencias transmedia y orientaciones didácticas para el uso y desarrollo de estas. El acceso a las herramientas es muy sencillo, además las herramientas no son lo primero ni lo más importante para aprovechar las competencias digitales en los aprendizajes formales. El punto de partida para innovar los 
procesos de enseñanza-aprendizaje es la comprensión y valoración del potencial educativo de estas competencias y herramientas.

\section{RESULTADOS}

Es así como:

Los estudiantes de entre 12 y 17 años evidencian capacidades de búsqueda y uso de información por medio de diferentes dispositivos y con diferentes finalidades.

Los estudiantes de entre 12 y 17 años utilizan tutoriales de YouTube para aprender, por un lado, situaciones de gusto y necesidad personal y, por otro lado, situaciones específicas de aprendizaje formal de su escolaridad. Los tutoriales de YouTube, incluso en algunos casos, son el principal medio de consulta incluso antes que sus docentes o padres.

Los adolescentes y jóvenes están aprendiendo, en espacios informales y ubicuos, por sus propios medios y con sus propias culturas educativas, en las que predomina la tutorización, tanto para el acceso como para la producción y publicación de recursos de aprendizaje transmedia.

La tutorización del YouTube se ha convertido en la estrategia más potente para el aprendizaje autónomo, ubicuo e informal; está llevando a adolescentes y jóvenes, del acceso y consumo de información a la producción y publicación de productos transmedia, en la lógica y la dinámica de la web 2.0.

\section{DISCUSIÓN Y CONCLUSIONES}

Las capacidades de gestión de los recursos transmedia van desde el uso de diferentes dispositivos de acceso a los recursos transmedia, pasando por el manejo de una diversidad de aplicaciones y buscadores, para llegar a las diferentes finalidades de acceso orientadas, no solo al ocio y esparcimiento sino también enfocadas al uso de estos recursos transmedia para resolver situaciones generadas en su vida escolar.

Los tutoriales de YouTube son un medio muy efectivo y de uso común, pueden ser utilizados por estudiantes de casi todas las edades. Su utilidad va desde las situaciones cotidianas y personales a las necesidades escolares.

Las capacidades, herramientas y narrativas transmedia constituyen una tendencia de aprendizaje informal y altamente efectivo.

El rol tutorial ha sido asumido y desarrollado al máximo por YouTube, convirtiéndose en una verdadera escuela al momento de aprender para entretenerse y resolver tópicos del mundo personal de adolescentes y jóvenes.

Para que las herramientas transmedia lleguen a las aulas como aliados estratégicos, primero hay que comprender su potencial educativo y su real presencia en los aprendizajes informales y, luego, recurrir a las mismas para aprender a usarlas. El uso de tecnología se aprende con tecnología. 


\section{REFERENCIAS BIBLIOGRÁFICAS}

Álvarez, F., Pérez, A., Prats, J., Didriksson, A. y Quinn, H. (2015). Modelo pedagógico de la UNAE. Azogues, Ecuador: UNAE.

Badia, M., Clariana, M., Gotzens, C., Cladellas, R. y Dezcallar, T. (2015). Videojuegos, televisión y rendimiento académico en alumnos de primaria. Píxel-Bit. Revista de Medios y Educación, 46, 25-38. DOI: http://dx.doi.org/10.12795/pixelbit.2015.i46.02

Betancur, S., Carmona, L., Contreras, R., Karam, J., Maestre, N., Romero, Y. y Uribe, S. (2014). Videojuegos y tic como Estrategias Pedagógicas: Formación para el uso seguro de internet. Cultura, Educación y Sociedad, 91-107. Recuperado el 10 de enero de 2019 desde http:// repositorio.cuc.edu.co/handle/11323/3075

Bolivar, W., Chaverra, D. y Monsalve, M. (2015). Argumentación y uso de aplicaciones web 2.0 en la Educación Básica. Revista Lasallista de Investigacion, 12(1), 58-64. Recuperado el 18 de julio de 2019 desde http://www.redalyc.org/pdf/695/69542290006.pdf

Cancelas, L. y Herrera, J. (2014). Reflexiones sobre el potencial de YouTube en la enseñanza y aprendizaje de una lengua extranjera. Educación y Comunicación, 11. Recuperado el 15 de febrero de 2019 desde https://www.researchgate.net/publication/318722477_Reflexiones_ sobre_el_potencial_de_YouTube_en_la_ensenanza_y_aprendizaje_de_una_Lengua_Extranjera

Gray, D., Brown, S. y Macanufo, J. (2012). Gamestorming: 83 juegos para innovadores, inconformistas y generadores del cambio. Bilbao: Deusto Ediciones.

Gutiérrez, J. y Gómez, M. (2015). Influencia de las TIC en los procesos de aprendizaje y comunicación de los estudiantes de educación. Revista de Pedagogia, 35-36(97-98), 34-51. Recuperado el 20 de enerode2019desdehttps://idus.us.es/xmlui/bitstream/handle/11441/33513/Influencia\%20de\%20 las $\% 20 \mathrm{TIC} \% 20 \mathrm{en} \% 201 \mathrm{os} \% 20$ procesos $\% 20 \mathrm{de} \% 20$ aprendizaje $\% 20 \mathrm{y} \% 20$ comunicación $\% 20$ de $\% 201$ os\%20estudiantes\%20de\%20ducación.pdf?sequence=1\&isAllowed=y

León, L. (2018). Niños YouTubers y el proceso de creación de videos : evidencia de competencias transmedia en acción. Comunicación y Sociedad, 33, 115-137. Recuperado el 18 de julio de 2019 desde http://www.comunicacionysociedad.cucsh.udg.mx/index.php/comsoc/article/view/7080/

Marín, V. (2015). La Gamificación educativa. Una alternativa para la enseñanza creativa . Digital Education Review, 27. Recuperado el 22 de marzo de 2019 desde http://revistes.ub.edu/index. $\mathrm{php} / \mathrm{der} / \mathrm{article} / \mathrm{view} / 13433 / \mathrm{pdf}$

QSR International. (2017). NVivo primeros pasos. México. Recuperado el 20 de febrero de 2019 desde http://download.qsrinternational.com/Document/NVivo11/11.4.0/es-MX/NVivo11Getting-Started-Guide-Pro-edition-Spanish.pdf

Robaina, N., Jenaro, C., González, F., Martín, E. y Poy, R. (2013). Adicción al móvil en alumnos de secundaria: efectos en la convivencia. European Journal of Investigation in Health, Psychology and Education, 3(3), 215-225.

Rodríguez, A., Hinojo, M. y Ágreda, M. (2017). Análisis del uso de video-tutoriales como herramiento de inclusión educativa. Publicaciones, 47, 13-35. Recuperado el 13 de mayo de 2019 desde https://revistaseug.ugr.es/index.php/publicaciones/article/view/7195

Ruiz, F. y Escurra, L. (2013). Hábitos de consumo de Facebook y YouTube. Conciencia y estrategias metacognitivas en la lectura y estrategias de aprendizaje y estudio en universitarios. Persona, 16, 29 - 71. Recuperado el 25 de junio de 2019 desde http://www.redalyc.org/ pdf/1471/147131896002.pdf

Salas, J. (2017). La OMS reconoce el trastorno por videojuegos como problema mental. El País, p. s.p. Recuperado el 27 de diciembre de 2018 desde https://elpais.com/elpais/2017/12/21/ ciencia/1513852127_232573.html

Siemens, G. (2004). Conectivismo: Una teoría de aprendizaje para la era. Licencia Creative Commons 2.5. Recuperado el 12 de enero de 2019 desde https://docs.google.com/document/d/1ZkuAzdx1191DgcC1E_XSmPTOk6Gu1K2SEvXtduG3gc/edit 
Scolari, C., Winocur, R., Pereira, S. y Barreneche, C. (2018). Alfabetismo transmedia. Una introducción. Comunicación y Sociedad, 33, 7-13. Recuperado el 25 de julio de 2019 desde http://www.comunicacionysociedad.cucsh.udg.mx/index.php/comsoc/article/view/7227/5998

Velarde, A., Dehesa, J., López, E. y Márquez, J. (2017). Los vídeo tutoriales como apoyo al proceso de enseñanza aprendizaje y sus implicaciones pedagógicas en el diseño instruccional. Educateconciencia, 14(15), 67-86. 
